\section{IDDF2021-ABS-0135 A RANDOMIZED CONTROLLED TRIAL COMPARING THE EFFECT OF LOW FODMAP DIETARY ADVICE AND NICE DIETARY ADVICE ON SYMPTOM CONTROL AND QUALITY OF LIFE IN CHINESE ADULTS WITH IRRITABLE BOWEL SYNDROME}

${ }^{1}$ Wing-yin Yeung* ${ }^{2}$ Geraldine Wai-bik Ng, ${ }^{3}$ Wing-han Cheung, ${ }^{4}$ Annie Ching-yan $N$ ${ }^{1}$ Edwin Hok-shing Shan, ${ }^{1}$ Chun-yu Say, ${ }^{1}$ Wai-chuen Wong, ${ }^{3}$ Elita Yun-nin Cheng, ${ }^{4}$ Ernest Han-fai Li, ${ }^{5}$ Tin-long Wong, ${ }^{6}$ Wai-hung Chow, ${ }^{2}$ Martin Ming-him Wong, ${ }^{1}$ Yiu-kay Chan, ${ }^{1}$ Department of Medicine and Geriatrics, Caritas Medical Centre, Hong Kong; ${ }^{2}$ Dietetic Department, Princess Margaret Hospital, Hong Kong; ${ }^{3}$ Dietetic Department, Caritas Medical Centre, Hong Kong; ${ }^{4}$ Private practice, Hong Kong; ${ }^{5}$ Department of Medicine and Therapeutics, The Chinese University of Hong Kong, Hong Kong; ${ }^{6}$ Department of Medicine, Yan Chai Hospital, Hong Kong

\subsection{6/gutjnl-2021-IDDF.154}

Background Western studies have shown the beneficial effect of low FODMAP (fermentable oligo-, di- and monosaccharides and polyols) diet on symptoms and quality of life (QOL) in patients with irritable bowel syndrome (IBS). There had not been a head-to-head comparison between low FODMAP dietary advice and the standard dietary advice by National Institute for Health and Care Excellence (NICE), focusing on how and when to eat, in IBS subjects in Chinese. This study aimed at comparing the efficacy of low FODMAP dietary advice versus NICE standard dietary advice in Chinese IBS subjects.

Methods This is a multi-center, prospective, open-label 2armed dietary intervention study. Seventy-two Chinese adults who fulfilled Rome III criteria for IBS were enrolled in three public hospitals in Hong Kong between October 2014 and June 2018. Each subject was randomized to receive the low FODMAP dietary advice (group A) or the NICE standard IBS dietary advice (2012 version) (group B) for 8 weeks. A 10point Likert scale symptom score (lower score indicating milder symptom) and IBS-QOL score (higher score indicating better QOL) were rated at baseline, week 4 and week 8. A mixed-effect model was used to compare the scores between both groups.

Results Four subjects in each group dropped out; sixty-four subjects completed the study (mean age 47.5 \pm 13.5, $59.4 \%$ female). Both groups demonstrated significant improvement in overall symptom scores (group A: from 5.94 to 3.91 to 3.72 ; group $\mathrm{B}$ : from 6.06 to 4 to 3.75 ; $P<0.001$ ), and IBS-QOL scores (group A: from 68.8 to 74.7 to 80.1 ; group B: from 64.8 to 71.6 to 75.5 ; $P<0.001)$ throughout the period. However, there was no significant difference between groups on both overall symptom scores $(P=0.990)$ and IBS-QOL score $(P=0.717)$. The proportions of subjects improved were similar in both groups $(P=\mathrm{NS})$. Sensitivity analysis, including all recruited subjects, showed a similar result.

Conclusions Both low FODMAP diet and NICE IBS diet are effective in improving symptoms and QOL scores in Chinese IBS adult subjects.

\section{IDDF2021-ABS-0139 THE ROLE OF ENDOSONOGRAPHY IN THE DIAGNOSIS OF HIATUS HERNIA}

Dmitriy Lukanin*, Alexey Sokolov, Alexey Sokolov, Irina Gritskova. Pirogov Russian National Research Medical University of the Ministry of Healthcare of the Russian Federation, Moscow, Russia
Background The leading method of diagnosing hiatus hernia $(\mathrm{HH})$ is esophagogastroduodenoscopy (EGDS) and contrast Xray examination of the esophagus and stomach. We've studied the possibilities of endoscopic ultrasonography (EndoUS) related to the diagnosis of $\mathrm{HH}$.

Methods EGDS, contrast X-ray and endoscopic ultrasonography were performed in 43 patients with clinical signs of $\mathrm{HH}$ requiring surgical correction. After receiving the results of the intraoperative revision, we evaluated the informative value of each of the methods.

Results According to the results of the intraoperative revision, $\mathrm{HH}$ type I was detected in $30(69,8 \%)$ patients, type III in $12(27,9 \%)$ and type IV in $1(2,3 \%)$ (a greater omentum was fixed in the hernial sac). Assessing the EGDS informative value showed that endoscopic signs of $\mathrm{HH}$ type $\mathrm{I}$ were detected only in $23(76,7 \%)$ patients, type III in $12(100,0 \%)$. The endoscopy misdiagnosing of axial hernias type I are due to the small size of the hernial sac and difficulties in assessing the position of gastroesophageal junction about the projection of the crura of diaphragm. Performing esophagogastroduodenoscopy doesn't provide reliable information about the presence of $\mathrm{HH}$ type IV. The contrast Xray made it possible to diagnose $\mathrm{HH}$ type $\mathrm{I}$ in $26(86,7 \%)$ patients, type III in $12(100,0 \%)$ and couldn't reliably determine the presence of $\mathrm{HH}$ type IV. Sometimes it is impossible to make a differential diagnosis between a hernia and a physiological esophageal distensibility in its supradiaphragmal site in small-sized $\mathrm{HH}$. The patient with $\mathrm{HH}$ type IV had no reliable radiological signs of the presence of a greater omentum in the postmediastinum. Performing EndoUS revealed signs of $\mathrm{HH}$ type I, III and IV in $100 \%$ of cases (IDDF2021-ABS-0139 Figure 1. Endoscopic ultrasound of hiatal hernia type I. 1. Heart; 2. Aorta; 3. Gastroesophageal junction) (IDDF2021-ABS-0139 Figure 2. Endoscopic ultrasound of hiatal hernia type III. 1. Heart; 2. Aorta; 3. Gastroesophageal junction; 4. Gaster). In the case of $\mathrm{HH}$ type I, including small ones, reliable signs of movement of the gastroesophageal junction above the level of the diaphragm were obtained in all patients.

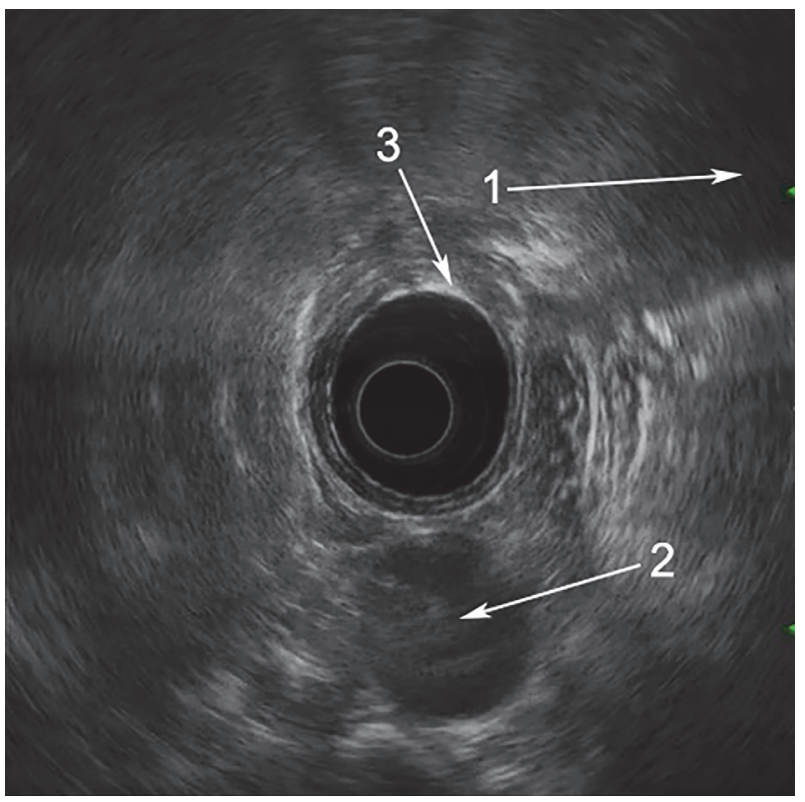

Abstract IDDF2021-ABS-0139 Figure 1 


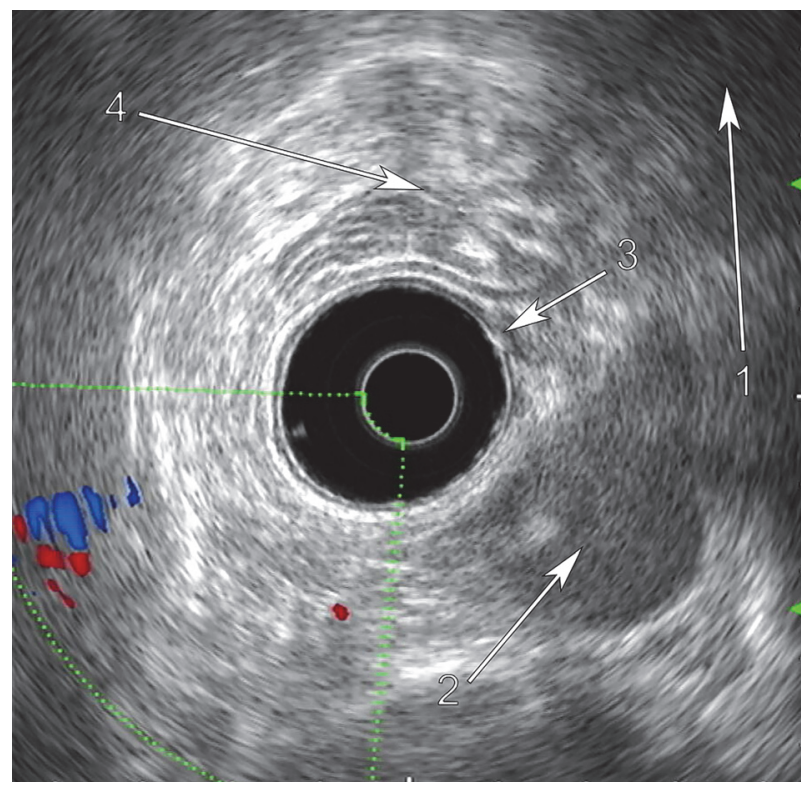

Abstract IDDF2021-ABS-0139 Figure 2

Conclusions Endoscopic ultrasonography has demonstrated high diagnostic value related to various anatomical variants of $\mathrm{HH}$. EndoUS is of the greatest importance for the verification of $\mathrm{HH}$ type I of small size.

\section{IDDF2021-ABS-0144 DENTAL INJURY DURING ENDOSCOPY: AN UNDERRECOGNIZED TEETHING SAFETY ISSUE}

${ }^{1}$ Gabrielle Loh Yi Wen*, 'Chelsea Tan Qiu Lin, 'Benjamin Tay Wei Rong, ${ }^{2} J o h n$ Mok, ${ }^{2}$ Calvin Koh Jianyi, ${ }^{2}$ Leo Juanda, ${ }^{2}$ Tien Ho Siah. ${ }^{1}$ National University of Singapore, Singapore; ${ }^{2}$ National University Hospital, Singapore

\subsection{6/gutjpl-2021-IDDF.156}

Background Dental injury is an underrecognized preventable complication during endoscopy. An audit of same-day procedure cancellation in our endoscopy center showed that $43 \%$ of cases are related to tooth safety concerns. A review of the literature showed that dental injury was under-reported in endoscopy related journals but an important issue in anaesthesia. Dental injury in anaesthesia is variably estimated to occur at a rate of approximately one in 1,000 to 4,500 . It represents up to half of all anaesthetic claims. We reviewed the preventive measures and management strategy of dental injury.

Methods We have reviewed the available literature and worked with a dental expert to suggest a few steps to improve tooth safety during endoscopy:

Results Key steps in preventive measures: 1) Pre-endoscopic tooth screening: Optimal pre-endoscopic assessment should include a comprehensive dental assessment, documentation and explanation of individualized risk involved. 2) Identification of risk factors: Evidence of periodontal disease and its infective complications, pre-existing dental pathology such as missing or loose teeth, and dental restorations. 3) Comprehensive documentation of pre-existing dental defects prior to endoscopy should be done.
Key steps in dental injury management: 1) Early involvement of dentist: If a dental injury occurs or a missing tooth is found during endoscopy, a dentist should be called to do an on-table consult. 2) Tooth retrieval whenever possible: If the dentist is unavailable or delayed, the missing tooth should be localised and retrieved. Upon retrieval of the tooth or tooth fragments, the dental officer on-call should be consulted for specific management. 3) Radiographic assistance: If localisation is not possible, a chest X-ray should be done, with the consultation of Thoracic Surgery.

Conclusions We encourage these simple measures to be implemented in the clinic and endoscopy center to prevent dental injury during endoscopy.

\section{IDDF2021-ABS-0146 PEDIATRIC COLONOSCOPY IN A TERTIARY CENTER: A 10-YEAR EXPERIENCE}

Jan Pofer Mantos*, Portia Menelia Monreal, Caroline Anne Castro. University of Santo Tomas Hospital, Philippines

\subsection{6/gutjnl-2021-IDDF.157}

Background There has been no retrospective study yet on the role of colonoscopy in children in our local setting; thus, this is the first study to review the experience of pediatric colonoscopy in the Philippines.

Methods This is a cross-sectional study through retrospective chart review with an analytical component. The study included in-patients aged 0 to 18 years old who underwent their first colonoscopy between January 1, 2010 and December 31, 2019 at the University of Santo Tomas Hospital.

Results A total of 196 respondents were included in the study with a mean age of 8.6 years, with slight male predominance $(51.5 \%)$.

Table 1. The most common indication for colonoscopy was hematochezia. Among the patients with hematochezia, 94.2\% had positive colonoscopy findings (IDDF2021-ABS-0146 Table $1)$.

Table 2. The most common findings were non-specific colitis, polyps, non-specific pancolitis, internal hemorrhoids, and ileitis (IDDF2021-ABS-0146 Table 2).

Table 3. The most common bowel preparation used were Fleet enema, Bisacodyl suppository, and Castor oil (IDDF2021-ABS-0146 Table 3).

Abstract IDDF2021-ABS-0146 Table 1 Descriptive Statistics for the Indications for Colonoscopy $(\mathrm{N}=196)$

\begin{tabular}{llll}
\hline $\begin{array}{l}\text { Indications of } \\
\text { Colonoscopy }\end{array}$ & $\begin{array}{l}\text { Frequency } \\
(f)\end{array}$ & $\begin{array}{l}\text { Percentage } \\
(\%)\end{array}$ & $\begin{array}{l}95 \% \text { Confidence Interval } \\
(\mathrm{Cl})\end{array}$ \\
\hline Hematochezia & 156 & $79.6 \%$ & $73.3 \%-84.7 \%$ \\
Constipation & 5 & $2.6 \%$ & $1.1 \%-6.0 \%$ \\
Melena & 10 & $5.1 \%$ & $2.8 \%-9.3 \%$ \\
Diarrhea & 10 & $5.0 \%$ & $2.8 \%-9.3 \%$ \\
Abdominal Pain & 12 & $6.1 \%$ & $3.5 \%-10.5 \%$ \\
Weight Loss & 1 & $0.5 \%$ & $0.1 \%-3.6 \%$ \\
Occult Bleeding & 1 & $0.5 \%$ & $0.1 \%-3.6 \%$ \\
Surveillance & 1 & $0.5 \%$ & $0.1 \%-3.6 \%$ \\
\hline
\end{tabular}

